\title{
Development of Reflective Thinking through Distance Teacher Education Programs at AIOU Pakistan
}
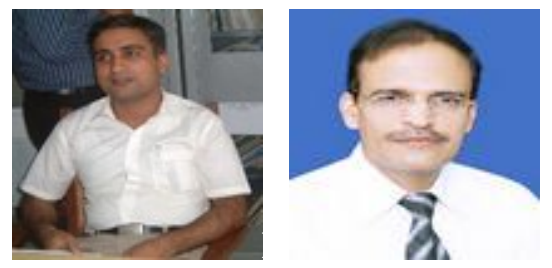

Muhammad Ayub Buzdar and Akhtar Ali

The Islamia University of Bahawalpur, Pakistan

\section{Abstract}

The current study aims to investigate the possibilities of developing reflective thinking among learners through distance education programs. The case of Allama Iqbal Open University (AIOU) Islamabad, Pakistan is examined to achieve this task. The study is based on Mezirow's theory of reflective thinking, which divides thinking in four categories. The Questionnaire of Reflective Thinking (QRT) developed by Kember et al. (2000) based on Mezirow (1991) was administered to 450 AIOU students. The thinking and learning practices of students governed by their habitual actions, understanding, reflection, and critical reflection are statistically examined to meet the research objectives. Findings reveal that AIOU teacher education programs have a stronger tendency to promote understanding and reflective thinking among learners. The need to integrate content for developing critical reflection among learners is highlighted. The study also discovers the significant impacts of students' job status and previous qualifications on their thinking patterns.

Keywords: Habitual action; understanding; reflection; critical reflection 


\section{Introduction}

Developing reflective thinking among learners is a fundamental aim of modern education. Dewy (1933, p. 9) defines reflection as "active, persistent and careful consideration of any belief or supposed form of knowledge in the light of grounds that support it and the further conclusions to which it tends." Schon (1995) and Rodgers (2002) make additions to Dewy's perspective offering the concept of "reflection in problem-solving." Reflection in problem-solving involves rethinking and reconsiderations of past events and experiences with the intention of gaining better and more refined solutions in future. Mezirow (1991) is more elaborative in defining reflection. He asserts that making "meaning" of any experience includes sensing and interpreting. Learning refers to the subsequent use of these meanings in decisionmaking. Mezirow hypothesizes that "meaning schemes" generate individuals' habitual actions which form and govern people's perspectives, assumptions, and decisions. People face different challenges including epistemic, psychic, and socio-cultural distortions, while generating and governing perspectives and decisions. Reflection, in Mezirow's view, refers to correct these procedural distortions whereas "critical reflection" challenges bases and presuppositions of generated "meanings" and "perspectives". Kember et al. (2000) derive four major states of thinking from Mezirow's theory, that is, habitual actions, understanding, reflection, and critical reflection.

Dinkelman (2000) determines that in educational setup, a teacher's role is more crucial and multi-dimensional in promoting reflective thinking. She has responsibility to protect her decisions from distortions as well as assess and reassess the basics of her academic decisions and instructional practices. Takona (2002) furthers that she evaluates students' attaining of thinking expertise and documents their school record. It is also her responsibility to sharpen reflection and critical reflection among students so that they can perform effectively in their future lives.

The question of how effectively teacher education institutions are preparing reflective teachers is gaining importance in contemporary research. Universities and teacher education institutions are attempting to make their programs more conducive for creating reflective thinking skills among graduates. In Pakistan, teacher education programs are offered at different levels. Allama Iqbal Open University (AIOU) Islamabad offers a variety of teacher education programs through postal correspondence, the Internet, radio, and television broadcasting. Curricular designs of AIOU teacher education programs comprise assignments, tutorial meetings, and workshops. Students are bound to submit two assignments and attend two day workshops (accumulatively 10 - 12 teaching hours) for each three credit hours course. Students are lectured basic concepts and contents of the courses in workshops. The current study aims to assess the presence of reflective thinking among prospective teachers getting training at AIOU Pakistan and examine the role of concerned syllabi in developing the four levels of thinking among student teachers. 


\section{The Current Study}

The present research established its foundations on Mezirow's theory of reflective thinking. Mezirow asserts three major kinds of thinking, that is making meaning (schemes and perspectives), reflection, and critical reflection. He adds that making meaning is the result of habitual expectations (that are based on previous experiences) or understanding (assimilation and interpretation of new experiences). In the current study we evaluated how the AIOU teacher education syllabi promoted the four thinking categories (i.e., habitual actions, understanding, reflection, and critical reflection). Examining the impacts of students' previous education and employment status on different phases of their thinking was also accepted as a supplementary objective of the study.

The AIOU Pakistan is among the largest universities in the world according to active enrollments. Development of reflective thinking is an essential objective of education. It gains more importance in the case of teacher education programs due to the significant and diversified role of teachers in educational organizations. A case study of AIOU strengthens the tradition of evaluating university programs on the basis of their potential to promote higher order thinking among students.

\section{Research Questions}

The following research questions were formulated to facilitate data analysis and achieve the research objectives.

1. How cognitively do AIOU students deal with their study programs?

2. To what extent are AIOU teacher education programs supportive in developing reflective thinking among learners?

3. What are the impacts of students' previous qualifications and employment status on their thinking choices?

\section{Research Methodology}

An inquiry of two existing phenomena (students' thinking practices and the role of the AIOU teaching-learning process in promoting these practices) was the focus of this research. Viewing the non-experimental descriptive nature of the study, a survey method was adopted to collect the required information (J ohnson \& Christenson, 2010). The AIOU offers undergraduate, graduate, and postgraduate teacher education programs with multiple entry and exit points. These programs prepare teachers to educate students from primary to university level. Students of undergraduate and graduate teacher education programs are trained to teach primary, elementary, and secondary classes. However graduates of postgraduate courses (Master of Education 
and Master of Arts in Education) are eligible to teach from primary to university level. The current study preferred to concentrate on the AIOU postgraduate courses of teacher training viewing the diversity of their curricula and the significance for the Pakistani educational system.

Multistage sampling was used to select the sample. Researchers selected nine out of 36 country wide AIOU regional centers as a convenience sample located at districts Bahawalpur, Rahim Yar Khan, Multan, Dera Ghazi Khan, Sargodha, J hang, Sahiwal, Miawali, and Attock. From each regional center, fifty students attending workshops of their terminal semesters were randomly selected $(n=9 \times 50=450)$. Four hundred and twenty-three future teachers returned the filled instruments. Although a sample size of 200 is considered to be reasonable for descriptive, correlational, and regression research designs a standard error of the mean (SEM) was calculated to estimate how the mean of the sample is related to the mean of the underlying population (Fraenkel \& Wallen, 2007; Kelley \& Maxwell, 2003; Kenny, 2011; Nagele, 2003). The mean score of the ages of 399 respondents was 29.09 years; the remaining 24 students did not give their ages. Age of the respondents ranged from 20 to 48 years. Sixty-four percent of the respondents were female.

A major limitation of the current study is the generalizability of its findings. Since the respondents were selected from nine regional centers of AIOU, statistical conclusions of the current study were applicable to the student teachers attending workshops of their terminal semesters in these centers. Generalization of the research findings to the student teachers attending workshops at other regional centers is not possible.

Development of a valid and reliable research instrument on the basis of Mezirow's theory of reflective thinking was the most important phase of the current study. A review of the related literature, however, demonstrated that the Questionnaire for Reflective Thinking (QRT) developed by Kember et al. (2000) was relevant, valid, and reliable to meet the objectives of the current research. The QRT is based on the writings of Mezirow (1991). Kember et al. claim that the four subscales of the QRT assess four levels of reflective thinking (i.e., habitual action, understanding, reflection, and critical reflection). Characteristics of each level of thinking as expressed by Kember et al. are described here :

1. Habitual action: Activity that is learnt through frequent use. Later, it is performed automatically or with little conscious thought.

2. Understanding: Thoughtful activity of individuals in which they use their existing knowledge and get comprehension of different things/phenomena. Kember et al. (2000) elaborate that the construct "understanding (U)" comprises an understanding of a concept without reflecting upon its significance in personal or practical situations.

3. Reflection: The critique and appraisal of assumptions about the content or process of problem solving. It differentiates problem posing from 
problem solving and raises questions regarding the validity of the problems' solutions.

4. Critical reflection: This is a profound level of reflection. Kember et al. elaborate that it involves testing of a premise. It reviews reflection evidence on the bases of conscious and unconscious prior learning. This level of reflective thinking is not observed frequently.

Kember et al. reported the values of Cronbach alpha as 0.621, 0.757, 0.631, and 0.675, respectively, for the constructs habitual action (HA), understanding (U), reflection (R), and critical reflection (CR). However the current study demonstrated Chronbach alpha values of $0.610,0.632,0.690$, and 0.741 for the constructs respectively. Standard errors of the means (SEMs) for the four subscales were estimated at $0.023,0.021,0.021$, and 0.025 relatively, which reveals strong narrowness between the sample and the underlying population means for the constructs. A brief introduction of the study, research objectives, scale, and subscales including the four levels of thinking was delivered to the respondents before administering the tool. The scale was administered to the sampled students converting it from a five to a four point Likert Scale from strongly disagree to strongly agree. Data were collected in the spring semester 2012.

\section{Findings}

Findings reveal that distance learners of AIOU teacher education programs support the idea that if they follow the lecturers and remember handout material, they do not have to think too much. In contrast, the learners oppose the statements that in their course they do things so frequently that they start to do these activities without thinking about it and they have a habit of doing so. The mean score of 2.36 for the construct "habitual thinking" demonstrates that, accumulatively, the AIOU learners have less attention to depend on their habitual actions (Table 1). 
Table 1

Students' Thinking Practices Governed by Habitual Actions

Sr. \# Statement

Mean

01 When I am working on some activities, I can do them without thinking 1.90 about what I am doing.

02 In this course, we do things so many times that I started to do them 1.77 without thinking about it.

03 As long as I can remember handout material for examinations, I do not 2.63 have to think too much.

04 If I follow what the lecturers say, I do not have to think too much on 3.15 this course.

Habitual action

Data further show that the distance learners support the idea of promoting AIOU students' understanding through their studies. Mean scores of 3.43, 3.57, 3.35, and 2.99 for the four statements of the construct "understanding" reveal the respondents' strong agreement in this regard (Table 2). The four statements demonstrate that the AIOU teacher education program requires understanding of the course concepts and study materials. Understanding is also considered necessary for success in examinations. The mean score representing the students' responses on the entire construct "understanding" is 3.34. It supports the notion that AIOU teacher education programs are conducive for generating understanding of content among the learners. 
Table 2

Students' Thinking Practices to Gain Understanding

Sr. \# Statement

Mean

01 This course requires us to understand concepts taught by lecturers.

3.43

02 To pass this course I need to understand the contents.

03 I need to understand the material taught by the lecturer in order to 3.35 perform practical tasks.

04 In this course I have to continuously think about the material I am 2.99 being taught.

Understanding

Findings demonstrate that the mean scores of students' responses on the four statements of the construct "reflection" are 3.07, 3.33, 3.47, and 3.63 (Table 3). It shows that distance learners of the AIOU teacher education programs have strong intentions to question the procedural process of problem solving and decision making adopted by themselves or others. A majority of them rethink and re-appraise their previous experiences with the objective to learn and improve their thinking practices. The mean score revealing the respondents' reflection is 3.63. It shows a strong reflective attitude among the prospective teachers studying under the AIOU distance learning program. 
Table 3

Students Thinking Practices Governed by Reflection

Sr. Statement Mean

\#

01 I sometimes question the way others do something and try to think of a 3.07 better way.

02 I like to think over what I have been doing and consider alternative way 3.33 of doing it.

03 I often reflect on my actions to see whether I could have improved on 3.47 what I did.

04 I often re-appraise my experience so I can learn from it and improve my 3.63 next performance.

Reflection

Table 4 shows the mean scores of 3.13, 2.94, 3.08, and 3.13 for the four statements of the construct "critical reflection", indicating that the AIOU teaching course supports the learners to challenge their firmly held ideas and help them to discover their underlying faults. The learners accept that they changed their normal way of doing things as a result of this course. Accumulative mean score of the construct "critical reflection" is 3.07 , which reveals that a majority of the students possesses critical reflection. 
Table 4

Students' Thinking Practices to Gain Critical Reflection

\begin{tabular}{llc}
\hline $\begin{array}{l}\text { Sr. } \\
\#\end{array}$ & Statement & Mean \\
\hline 01 & As a result of this course, I have changed the way I look at myself. & 3.13 \\
02 & This course has challenged some of my firmly held ideas. & 2.94 \\
03 & As a result of this course I have changed my normal way of doing things. & 3.08 \\
04 & $\begin{array}{l}\text { During this course I discovered faults in what I had previously believed } \\
\text { to be right. }\end{array}$ & 3.13 \\
\hline & Critical reflection & 3.07 \\
\hline
\end{tabular}

Comparison among the student teachers' responses in the four subscales demonstrates that the majority of them avoid making decisions through habitual actions. Data reveal that the assertion to use reflection and understanding in curricular designs of AIOU teacher education programs is stronger than promoting habitual actions and critical reflection (Table 5). The students avoid making decisions on the basis of habitual action whereas, to some extent, they support critical reflection. The presence of critical reflection among the distance learners is lower than reflection and understanding.

Table 5

Presence of Four Levels of Thinking in AIOU Learners

\begin{tabular}{lllll}
\hline & Habitual action & Understanding & Reflection & $\begin{array}{l}\text { Critical } \\
\text { reflection }\end{array}$ \\
\cline { 2 - 5 } Mean scores & 2.36 & 3.34 & 3.38 & 3.07 \\
\hline
\end{tabular}

The AIOU offers distance learning programs to employed and unemployed learners. To find out the impacts of students' employment status on their thinking practices, $t$ test was applied. Findings demonstrate that the t values of -3.248, -3.320, and -3.964 for habitual action (HA), understanding (U), and critical reflection (CR) respectively are significant at the level of 0.001 (Table 6). However the $t$ value for the construct reflection $(\mathrm{R})$ is insignificant. 
Table 6

Impact of AIOU Students' Employment Status on their Thinking Patterns

\begin{tabular}{|c|c|c|c|c|c|c|}
\hline Subscale & Status & $\mathrm{N}$ & Mean & $\mathrm{t}$ & M. D. & d \\
\hline \multirow[t]{2}{*}{ Habitual action } & Employed & 300 & 2.32 & $-3.248 *$ & -0.167 & 0.73 \\
\hline & Unemployed & 123 & 2.48 & & & \\
\hline \multirow[t]{2}{*}{ Understanding } & Employed & 300 & 3.30 & $-3.320 *$ & -0.144 & 0.72 \\
\hline & Unemployed & 123 & 3.44 & & & \\
\hline \multirow[t]{2}{*}{ Reflection } & Employed & 300 & 3.37 & -0.447 & -0.021 & 0.18 \\
\hline & Unemployed & 123 & 3.39 & & & \\
\hline \multirow[t]{2}{*}{ Critical reflection } & Employed & 300 & 3.02 & $-3.964 *$ & -0.190 & 0.73 \\
\hline & Unemployed & 123 & 3.21 & & & \\
\hline
\end{tabular}

Note: $\mathrm{N}$ = sample size, $\mathrm{t}=\mathrm{t}$ value, $\mathrm{M}$. $\mathrm{D}$. = mean difference, $\mathrm{d}=$ = Cohen's d (effect size)

*the value is significant at the level of 0.001

Values of Cohen's d, representing the impacts of students' employment status on their thinking patterns, are $0.73,0.72$, and 0.73 for habitual action (HA), understanding (U), and critical reflection (CR) respectively (Table 6). It reveals a medium effect size of students' employment status on the three subscales of QRT. The students who are unemployed are more active in habitual action (HA), understanding (U), and critical reflection (CR). However data do not reveal a significant impact of employability on students' reflection. 
Table 7

Impact of AIOU Students' Previous Education on their Thinking Patterns

\begin{tabular}{|c|c|c|c|c|c|c|}
\hline Subscale & $\begin{array}{l}\text { Previous } \\
\text { Education }\end{array}$ & $\mathrm{N}$ & Mean & $\mathrm{t}$ & M. D. & d \\
\hline \multirow[t]{2}{*}{ Habitual action } & B.A./ B.Sc. & 148 & 2.36 & -0.118 & -0.006 & 0.23 \\
\hline & M.A./M.Sc. & 275 & 2.37 & & & \\
\hline \multirow[t]{2}{*}{ Understanding } & B.A./ B.Sc. & 148 & 3.45 & 4.119* & 0.176 & 0.88 \\
\hline & M.A./M.Sc. & 275 & 3.27 & & & \\
\hline \multirow[t]{2}{*}{ Reflection } & B.A./ B.Sc. & 148 & 3.28 & $-3.704^{*}$ & -0.152 & 0.83 \\
\hline & M.A./M.Sc. & 275 & 3.43 & & & \\
\hline \multirow[t]{2}{*}{ Critical reflection } & B.A./ B.Sc. & 148 & 3.20 & $3.830^{*}$ & 0.184 & 0.71 \\
\hline & M.A./M.Sc. & 275 & 3.01 & & & \\
\hline
\end{tabular}

Note: $\mathrm{N}=$ sample size, $\mathrm{t}=\mathrm{t}$ value, $\mathrm{M}$. D. = mean difference, $\mathrm{d}=$ =Cohen's $\mathrm{d}$ (effect size)

*the value is significant at the level of 0.001

Findings reveal that the $t$ value showing the effect of students' previous education on their habitual actions is insignificant. However, the t values to demonstrate the impacts of previous education of distance learners on their understanding (U), reflection (R), and critical reflection (CR) are 4.119, -3.704, and 3.830. These values are significant at the level of 0.001. Values of Cohen's d, 0.88 and 0.83 , show that students' previous education has large size effects on their understanding and reflection, whereas the effect size of previous education on distance learners' critical reflection is medium as depicted by Cohen's $d$ value of 0.71 . The $t$ values and mean differences show that the impact of students' previous education is positive on the subscales of understanding (U) and critical reflection (CR), whereas it is negative on the scale reflection (R). 


\section{Discussion}

Findings reveal that the students accumulatively discourage the idea of using habitual actions in their study matters. Course requirements of the AIOU program and syllabi compel the students to work beyond using habitual actions in decision making. In previous research, Bakhsh (2010) investigated different components of the AIOU teacher education programs in detail. He concludes that according to a majority of learners, study materials and course content of AIOU teacher education programs are comprehensive and easily understandable. The current study verifies and further elaborates the results of Bakhsh. It reveals that the content of AIOU teacher education programs are not only self-exploratory and easily understandable, but they promote students' attitude of comprehension and reflection as well.

This study reveals that AIOU teacher education programs promote students' understanding and reflection more than develop critical reflection. Kember et al. (2000) conclude that the presence of critical reflection is, generally, lesser in people than understanding and reflection. The current study authenticates their findings. The AIOU teacher education programs highly support and promote reflection and understanding among future teachers. Hussain, Mehmood, and Sultana (2011) investigated the benefits of reflective practices in the open and distance learning system in Pakistan. Although they support the presence of reflective practices in distance study programs, the contribution of existing distance study programs in developing reflective thinking among learners is not comprehensively examined in their research. The current research fills this gap determining that the teacher education programs of AIOU discourage learners' attitudes of using habitual actions in their course requirements. Similarly, the programs are significant promoters of gaining understanding and using reflection to fulfill students' course requirements.

Impacts of students' employment status and previous education on their responses were examined to further explore the promotion of reflective thinking through AIOU teacher education programs. The employment status of the students has medium size effects on their habitual actions, understanding, and critical reflection. The impact of students' employment status on their reflection (R) is insignificant. Viewpoints of employed and unemployed learners are significantly varied on the three subscales of QRT. The unemployed learners demonstrate stronger agreement with the idea that their study courses promote understanding and critical reflection among learners. It can be assumed that unemployed learners can spend more time on their studies than their employed fellows. Consequently their perceptions about the role of AIOU courses in developing understanding and critical thinking of students are stronger. However the findings do not present a significant impact of the students' employment status on their perceptions about the development of reflection through the study programs. Promoting reflection among the students is a core objective of the AIOU distance education programs (Bakhsh, 2010). Opinions of employed and unemployed students are not significantly different on this important issue. 
Learners in the AIOU teacher education programs approached in the current study have two types of previous education. One cohort enters the programs after passing bachelor degrees (i.e., 14 years of education). The other group enrolls in the same AIOU teacher education programs after passing master degrees (i.e., 16 years of education). Results show that the learners' previous education has strong size impacts on their thinking practices and opinions regarding the role of AIOU teacher education programs in promoting understanding and reflection among the students. The study further elaborates that the effect size of learners' previous education on their perceptions about the development of critical reflection through AIOU teacher education programs is reportedly medium. Values of $t$ test and mean differences explain the directions of these effects. The students having 14 years of previous education have a stronger commitment to the idea of promoting understanding and critical reflection through the programs. However the situation is inversed for the subscale of reflection. Student teachers having 16 years of education have a stronger tendency to accept the AIOU teacher education programs as developers of reflective thinking among the students than their bachelor degree holder fellows. Overall and segment wise opinions of the respondents endorse that the AIOU teacher education programs are supporters and promoters of understanding, reflection, and critical reflection among the learners.

\section{Conclusions}

The current study examined the thinking patterns of AIOU teacher education learners and the role of their courses in developing these patterns on the basis of Mezirow's theory of reflective thinking. It is concluded that the AIOU teacher education syllabi discourage making decisions on the bases of habitual actions. The learners are also restricted in making meaning and interpreting new experiences unintentionally. The AIOU teacher education programs have a stronger tendency to promote understanding and reflection among the learners. Prospective teachers use deliberation in interpreting their new experiences and challenge procedural evidence of making meaning and perspectives. The study concludes that the AIOU programs have comparatively lower intentions to support critical reflection among learners than to promote understanding and reflection. Critical reflection, in Mezirow's theory, is the highest level of reflective thinking that challenges the fundamentals of individual decisions. It results, in many cases, in a paradigm shift. It is important that there is evidence of support for critical reflection in the AIOU programs yet their presence is lower than the content supporting understanding and reflection.

It is also concluded that students' employment status and previous qualifications have significant impacts on their thinking preferences. The unemployed students are comparatively stronger in their opinions that AIOU teacher education programs encourage reflective thinking among the learners. Similarly the students having 16 years previous education demonstrate a higher level of commitment to the idea of promoting reflective thinking through the AIOU programs. 
Investigation of AIOU teacher education programs, regarding their role in the development of reflective thinking among students, supports the programs' worth and effectiveness. A major contribution of the current study is its evidence-based authentication that distance learning programs can promote the development of reflective thinking among learners. The study also highlights the possibilities of improvements in the case of critical reflection. The programs, though, have the intention of promoting critical reflection among learners yet the presence of such material is lower than understanding and reflection-promoting material. The task of developing critical reflection is at the top in educational objectives. The current study recommends integrating content in distance education curricular designs that is strongly supportive in developing critical reflection among the learners especially in the AIOU context. 


\section{References}

AIOU. (n. d.). Institutional profile of Allama Iqbal Open University Islamabad Pakistan. Retrieved from http:// www.aiou.edu.pk/ salientfeatures.asp

Bakhsh, Q. (2010). A study of students perception regarding teacher education through distance learning in Pakistan. International J ournal of Digital Society, 1(1). Retrieved from http:// www.infonomicssociety.org/ IJ DS/ A\%20Study\%20of\%20Students\%20Perception\%20Regarding \%20Teacher\%20Education\%20through\%20Distance\%20Learning\%20in\%20P akistan.pdf

Dewey, J . (1933). How we think. Lexington, MA: D.C. Heath \& Co.

Dinkelman, T. (2000). An inquiry into the development of critical reflection in secondary student teachers. Teaching and Teacher Education, 16(2), 195-222.

Fraenkel, J . R., \& Wallen, N. E. (2007). How to design and evaluate research in education (6 $6^{\text {th }}$ ed.). New York: McGraw-Hill.

Hussain, M. A., Mehmood, A., \&Sultana, M. (2011). An inquiry into benefits of reflective practice in open and distance learning. Turkish Online J ournal of Distance Education, 12(2), 51-59. Retrieved from https://tojde.anadolu.edu.tr/tojde42/pdf/article_2.pdf

J ohnson, B., \& Christenson, L. (2010). Educational research: Quantitative, qualitative and mixed approaches. Thousand Oaks: Sage Publications.

Kelley, K., \& Maxwell, S. E. (2003). Sample size for multiple regression: Obtaining regression coefficients that are accurate, not simply significant. Psychological Methods, 8(3), 305-321.

Kember, D., Leung, D., J ones, A., Loke, A. Y., et al. (2000). Development of a questionnaire to measure the level of reflective thinking. Assessment and Evaluation in Higher Education, 25(4), 380-395.

Kenny, D.A. (2011). Measuring model fit. Retrieved from http:// davidakenny.net/ cm/ fit.htm

Mezirow, J . (1991). Transformative dimensions of adult learning. San Francisco: J ossey-Bass.

Nagele, P. (2003). Misuse of standard error of the mean (SEM) when reporting variability of a sample. A critical evaluation of four anaesthesia journals. British J ournal of Anaesthesia, 90(4), 514-516. doi : 10.1093/ bja/ aeg087 
Rodgers, C. (2002). Defining reflection: Another look at J ohn Dewey and reflective thinking. Teachers College Record, 104(4), 842-866.

Schon, D. A. (1995). The reflective practitioner: How professionals think in action. Farnham, UK: Ashgate Publishing.

Takona, J . P. (2002). Pre-service teacher portfolio development. Lincoln, NE: Writers Club Press.

\section{Athabasca University $\mathbf{A}$}

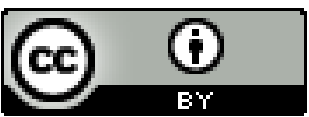

